\title{
Recurrent versus first cervical artery dissection - a retrospective study of clinical and vascular characteristics
}

Kloss, M.

2020-11

Kloss , M , Kalashnikova , L, Dobrynina , L, Traenka, C , Engelter , S T, Metso , T M , Tatlisumak , T , Urbanek , C , Grau , A , Kellert , L , Brandt , T , Wieker , C M , Grond-Ginsbach , C \& Pezzini , A 2020 , ' Recurrent versus first cervical artery dissection - a retrospective study of clinical and vascular characteristics ' , European Journal of Neurology , vol. 27 , no. 11 , pp. 2185-2190 . https://doi.org/10.1111/ene.14417

http://hdl.handle.net/10138/332516

https://doi.org/10.1111/ene.14417

acceptedVersion

Downloaded from Helda, University of Helsinki institutional repository.

This is an electronic reprint of the original article.

This reprint may differ from the original in pagination and typographic detail.

Please cite the original version. 
DR. LARS KELLERT (Orcid ID : 0000-0002-4967-8336)

DR. CASPAR GROND-GINSBACH (Orcid ID : 0000-0002-6882-3148)

DR. ALESSANDRO PEZZINI (Orcid ID : 0000-0001-8629-3315)

Article type : Original Article

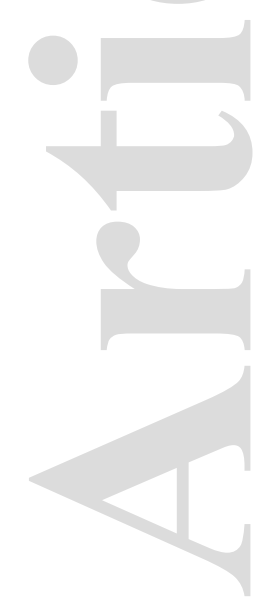

(

This article has been accepted for publication and undergone full peer review but has not been through the copyediting, typesetting, pagination and proofreading process, which may lead to differences between this version and the Version of Record. Please cite this article as doi: 10.1111/ENE.14417

This article is protected by copyright. All rights reserved 


\section{Recurrent versus first cervical artery dissection - A retrospective study of clinical and vascular characteristics.}

Manja Kloss, ${ }^{1}$ Ludmila Kalashnikova, ${ }^{2}$ Larissa Dobrinina, ${ }^{2}$ Christopher Traenka, ${ }^{3,4}$ Stefan T Engelter, ${ }^{3,4}$ Tiina M Metso, ${ }^{5}$ Turgut Tatlisumak, ${ }^{5,6}$ Christian Urbanek, ${ }^{7}$ Armin Grau, ${ }^{7}$ Lars Kellert, ${ }^{8}$ Tobias Brandt, ${ }^{9}$ Carola Marie Wieker ${ }^{10}$, Caspar Grond-Ginsbach, ${ }^{1}$ Alessandro Pezzini ${ }^{11}$

1. Department of Neurology, University of Heidelberg, Heidelberg, Germany

2. Research Center of Neurology, Russian Academy of Medical Sciences, Moscow, Russia

3. Department of Neurology and Stroke Center, Basel University Hospital, Basel, Switzerland

4. Neurology and Neurorehabilitation, University Department of Geriatric Medicine FELIX PLATTER, University of Basel, Switzerland

5. Department of Neurology, Helsinki University Central Hospital, Helsinki, Finland

6. Department of Clinical Neuroscience/Neurology, Institute of Neuroscience and Physiology, Sahlgrenska Academy at University of Gothenburg and Department of Neurology, Sahlgrenska University Hospital, Gothenburg, Sweden

7. Department of Neurology, Klinikum Ludwigshafen, Ludwigshafen, Germany

8. Department of Neurology, University Hospital, Ludwig Maximilian University Munich, Germany

9. Suva / Swiss National Accident Insurance Fund, Lucerne, Switzerland

10. Department of Vascular and Endovascular Surgery, University of Heidelberg, Heidelberg, Germany

11. Department of Clinical and Experimental Sciences, Neurology Clinic, University of Brescia, Italy

\section{Authors' contribution:}

Drs. Manja Kloss, Caspar Grond-Ginsbach, and Alessandro Pezzini had full access to all the data in the study and takes responsibility for the integrity of the data and the accuracy of the data analysis. Study concept and design: Manja Kloss, and Caspar Grond-Ginsbach. Acquisition of data: All authors. Interpretation of data: Manja Kloss, Caspar Grond-Ginsbach and Alessandro Pezzini. Drafting of the manuscript: Manja Kloss, Caspar Grond-Ginsbach and Alessandro Pezzini. Critical revision of the manuscript for important intellectual content: All authors. Data analysis: Manja Kloss, Caspar Grond-Ginsbach and Alessandro Pezzini. Statistical analysis: Manja Kloss, Caspar Grond-Ginsbach and Alessandro Pezzini. Administrative, technical, or material support: Alessandro Pezzini. Study supervision: Manja Kloss, Caspar Grond-Ginsbach and Alessandro Pezzini.

This article is protected by copyright. All rights reserved 


\section{Ethical approval and informed consent}

The study protocol was approved by relevant local authorities in all centers and complied with national regulations concerning ethics committee approval and informed consent. All patients gave written informed consent prior to study participation.

\section{Correspondence:}

Alessandro Pezzini

Dipartimento di Scienze Cliniche e Sperimentali

Clinica Neurologica

Università degli Studi di Brescia

P.le Spedali Civili, 1

25123 Brescia

Italia

Tel: +39.030 .3384086$

Fax: +39.030. 3384086

e-mail: ale_pezzini@hotmail.com

alessandro.pezzini@unibs.it

ORCID: 0000-0001-8629-3315 
Recurrent versus first cervical artery dissection - A retrospective study of clinical, vascular and personal characteristics.

Running title: recurrent versus first CeAD

Word count: 2,236

Abstract: 223

References: 19

The present manuscript includes 2 tables

Keywords: arterial dissection; epidemiology; cerebrovascular diseases and cerebral circulation; cerebral infarction 
Objective - Most recurrent cervical artery dissection (CeAD) events occur shortly after the acute first CeAD. This study compared characteristics of recurrent and first CeAD events and searched for associations between subsequent events of an individual person.

Methods - CeAD patients with a new CeAD event occuring during a three to six months followup were retrospectively selected in seven specialized stroke centers. Clinical and vascular characteristics of the initial and the recurrent CeADs were compared.

Results - The study sample included 76 patients. Recurrent CeADs were occlusive in one (1.3\%) patient, caused cerebral ischemia in 13 (17.1\%) and were asymptomatic in 39 (51.3\%) patients, compared to $29(38.2 \%)$ occlusive, $42(55.3 \%)$ ischemic and no asymptomatic first CeAD events. In 52 (68.4\%) patients, recurrent dissections affected both internal carotid arteries or both vertebral arteries, while 24 (31.6\%) patients had subsequent dissections in both types of artery. Twelve (28.6\%) of 42 patients with an ischemic first dissection had ischemic symptoms due to the recurrent CeADs, too. However, only one (1.3\%) of 34 patients with a non-ischemic first CeAD suffered ischemia upon recurrence.

Conclusion - Recurrent CeAD typically affects the same site of artery. It causes ischemic events less often than first CeAD. The risk that patients, who presented with solely non-ischemic symptoms of a first CeAD, will have ischemic symptoms in case of a recurrent CeAD seems very small. 


\section{Introduction}

Cervical artery dissection (CeAD) is a common cause of stroke in young patients [1]. CeAD may present with warning symptoms, like headache, cervical pain or Horner syndrome. After these warning symptoms, cerebral or retinal ischemia was reported to occur in most (50\% to $95 \%)$ cases $[1,2]$, though recent longitudinal studies indicate much lower stroke rates [3]. In most hospitalbased series, $60-80 \%$ of CeAD patients had stroke $[4,5,6]$. Most patients have a single CeAD during their life, but $10-15 \%$ of the patients present with multiple dissections in different cervical arteries during the acute phase, or with new dissection shortly after the first event [6,7], which suggests the existence of a transient vulnerable phase with increased risk of CeAD [8,9]. Although cohorts of patients with recurrent $\mathrm{CeAD}$ were relatively small so far, few recurrent dissections were observed to cause stroke [8,9]. Interestingly, in most patients with multi-vessel CeAD, stroke was confined to the vascular territory of a single artery, while additional dissections were often asymptomatic [10].

The present study analyzed a retrospective series of patients with early recurrent CeAD events. In all patients, we analyzed the symptoms, the arterial imaging findings (categorized as occlusion, stenosis or normal) and the dissection site (carotid artery or vertebral artery) of the initial and of the recurrent event. The aims of the study were to characterize and compare first and recurrent CeAD events and to test, whether recurrent CeAD causes ischemia less often than initial CeAD. A further aim of our study was to explore whether subsequent CeAD events of an individual patient were similar.

\section{Subjects and Methods}

\section{Patients}

Patients with a recurrent CeAD event occurring during early follow-up (i.e. within a follow-up period of 3-6 months) after acute CeAD were recruited in seven European Neurology Departments. The centers had retrospectively selected these patients from their registers of CeAD patients that were recruited between January 2006 and December 2016. Institutional Review Boards at each participating center approved the study and patients provided written informed consent. All patients underwent a three-to-six months vascular imaging follow up, including MRI with magnetic resonance angiography (MRA; 3-dimensional time of flight) and/or CT angiography of the brain and neck vasculature. The presence of the double-lumen sign, luminal narrowing with the "string sign," and gradual tapering ending in total occlusion of the lumen 
(flame-like occlusion) were considered reliable angiographic findings of $\mathrm{CeAD}$, whereas a narrowed lumen surrounded by a semilunar-shaped intramural hematoma on axial T1-weighted images was considered the pathognomonic MR sign [4]. Recurrent CeAD was defined in the same manner as initial CeAD.

\section{Variables}

CeAD events were classified based on 1) dissection site (internal carotid artery (ICA), vertebral artery (VA), or both); 2) clinical presentations (ischemia (TIA or ischemic stroke with or without local symptoms), local signs only (Horner syndrome, cervical pain, headache, tinnitus, caudal nerve palsy or other symptoms in the absence of ischemia), and no symptoms or signs at all), and

3) degree of lumen narrowing (complete occlusion, stenosis (50-99\% reduction of the vessel diameter) and normal flow (normal lumen or less than 50\% stenosis)). Variables were defined as described before [4]. Degree of lumen narrowing was encoded as categorical variable with stenosis $=2$, occlusion $=1$ and normal lumen $=0$ ).

\section{Aims}

The current study had the following aims: 1) to explore whether recurrent CeAD events present less often with cerebral ischemia than initial CeAD events; 2) to describe the arterial location and the category of lumen narrowing of recurrent CeAD events; and 3 ) to compare characteristics of initial and recurrent CeAD events within the same person.

\section{Statistical analyses}

Differences between groups were compared with Fisher-exact test, Chi-square test and Student ttests, as appropriate. Chi-square test, Student t-test and logistic regression analysis were performed using the SPSS (version 21.0) package (www.spss.com). For the Freeman-Halton extension of the Fisher's exact test, vassarstats calculators were used (http://vassarstats.net/fisher2x3.html and http://vassarstats.net/fisher3x3.html). Since six explorative tests were performed, resulting $p$ values of each test were multiplied by 6 to adjust for multiple testing. $P \leq 0.05$ on 2 -sided test was considered significant.

A logistic regression model was proposed to identify independent predictors of ischemia in the total sample of all CeAD events (all first events plus all recurrent events). The presence of ischemic symptoms was the dependent variable in the model. As predictors the following items 
were entered: age of onset of first CeAD (in years), sex ( $\operatorname{man}=1$, woman=2), vessel site (encoded as "at least one internal carotid artery affected $=1$; no carotid artery affected $=0$ ), multivessel dissection (multi-vessel $=1$, single-vessel $=0$ ), vascular pathology $($ occlusion $=2$, stenosis $=1$, normal luman=0) and temporal order of the events (first $\mathrm{Ce} A D=1$, recurrent $\mathrm{CeAD}=2$ ).

\section{Data availability statement}

The data that support the findings of this study are available from the corresponding author upon reasonable request.

\section{Results}

The study sample included 76 patients with a total of $152 \mathrm{CeAD}$ events (76 first and 76 recurrent events). All recurrent CeAD events were detected within a 3-6 months follow-up period with a vascular imaging examination at the end.

The characteristics of the first and recurrent events were compared in Table 1 (A, C, E). Fifty (65.8\%) patients had a first CeAD event in the internal carotid arteries (ICA). First events were occlusive in 29 (38.2\%) patients and caused ischemic symptoms in 42 (55.3\%) patients. Recurrent events located in the ICA in 37 (48.7\%) patients. Only a single (1.3\%) recurrent event was occlusive. Recurrent CeAD events caused ischemic symptoms in 13 (17.1) patients. Recurrent events were less often occlusive $(p<0.001)$ and caused fewer ischemic symptoms $(p<0.001)$ compared to first CeAD.

Associations between the first and the recurrent events of the same individual (within-patient associations) were visualized in $3 \times 3$ contingency tables (Table 1, B,D,F). A strong dependency was found between the sites of the first and second dissections of a same patient $(p=0.0008)$ : Thirty-two patients had subsequent ICA dissections and 20 had subsequent VA dissections (Table 1B). With a single exception, recurrent dissections occurred in the intact contralateral artery. Vascular pathology did not correlate significantly between the subsequent CeAD events of individual patients (Table 1D: adjusted Fisher exact test for contingency: $p=0.08$ ).

The symptoms of subsequent $\mathrm{CeAD}$ events showed significant within-patient correlation: In 12 of the 42 patients with initial ischemia, recurrent CeAD event caused again ischemia. On the 
contrary, only one of the 34 patients with a non-ischemic first CeAD suffered ischemia due to the recurrent event $(p=0.004)$.

With a logistic regression model we sought to identify determinants of ischemia due to the 152 CeAD events (76 patients with two CeAD events). Logistic regression analysis confirmed the association between ischemia and vessel pathology and furthermore revealed that first events were more likely to cause ischemia than recurrent events, independently from vessel pathology (Table 2).

\section{Discussion}

This retrospective analysis of first and recurrent CeAD events in 76 patients had the following key findings: 1) Recurrent CeAD events were rarely occlusive and caused less often ischemia than initial CeADs; 2) After adjustment for vascular imaging findings, recurrent CeAD events remained far less likely to cause ischemia, compared to first CeAD events; 3) first and recurrent dissections of the same patient often affected the same arterial system (either twice in the carotid or twice in the vertebral arteries).

These findings have an immediate clinical impact: If the first dissection of a patient caused ischemic symptoms, only about $1 / 4$ of these patients will have ischemic symptoms in case of a recurrent dissection. More importantly, in case, the initial CeAD did not cause ischemic symptoms, the likelihood, had a recurrent CeAD will cause a stroke or TIA seems very small.

Previous studies indicated that the risk of recurrent dissections is high during a short vulnerable period of a few weeks and decreases afterwards to about $1 \%$ per year [8,9]. The current investigation does not permit conclusions about the risk of recurrent dissection to occur, but it indicates that recurrent dissections - at least those occurring during a follow-up period of 3-6 months - are often asymptomatic and cause fewer strokes or TIAs than first CeAD events. A novel observation of the current study is that recurrent dissections are rarely occlusive. This finding remains unexplained so far. The finding is important since vessel occlusion is an important predictor for clinical severity and for unfavorable outcome of CeAD patients [11]. Logistic regression analysis yielded a further result of this study: After adjustment for the milder vascular imaging findings of recurrent $\mathrm{Ce} A \mathrm{D}$ events, recurrent $\mathrm{Ce} A \mathrm{D}$ remained less likely to cause ischemic symptoms (compared to first CeAD events) with an estimated adjusted odd ratio of 0.373. We suspect that recruitment in stroke centers may have led to an excess number of patients 
with ischemic first CeAD events. Our observations cast doubt that such a high proportion of stroke patients is representative for the whole CeAD population. In the current study sample, 42 (55.3\%) patients had a first CeAD event with ischemic symptoms. This observed frequency is probably not a representative risk estimate, all the more since it is an order of magnitude above the risks due to other types of acute carotid injury [12-15].All first and the recurrent CeADs were diagnosed in the same centers by the same physicians with the same methods during the same years. The different proportion of ischemic events between first and second events seems therefore indisputable.

It is a puzzling observation that stroke is not more frequent in patients with multi-vessel CeAD from large hospital-based registries, compared to those with single-vessel events. However, in a recent analysis of 30 patients with recurrent CeAD events, multivessel events caused more strokes than single-vessel CeAD [7]. The increased risk of multivessel CeAD, compared to single-vessel CeAD was apparently detectable in a small series of recurrent events, but remained unappreciated in large hospital-based samples.

Our study revealed similarities of subsequent CeAD events on the level of the individual person: In most patients, CeAD occurred either in both carotid arteries or in both vertebral arteries. Similarly, most patients with simultaneous multivessel CeAD had bilateral dissections of the internal carotid or of the vertebral arteries, whereas patients with dissections in both arterial systems were rare [7]. Moreover, family studies indicated concordance of dissection site within pedigrees [16].

In the current study sample, the risk of ischemia due to CeAD differed among individuals: those with a first $\mathrm{CeAD}$ causing ischemic symptoms had a risk for ischemia during a recurrent $\mathrm{CeAD}$ event which is higher than in patients who presented with non-ischemic symptoms for their initial CeAD. We can only speculate on the reasons. This personal risk for ischemic CeAD may be related to the individual collateral circulation [17] but genetic variation of the coagulation system, of the vascular frailty or of other systems may also play a role.

This study has several limitations. The analyzed sample of patients with early recurrent CeAD was the largest so far, but it was still too small to establish robust and definitive associations.

Moreover, the short delay between the previous and the recurrent CeAD event made it difficult to differentiate between the effect of trigger factors (like mechanical injury or infectious diseases) on each of the events. As a consequence, the role of trigger factors in the pathogenesis of recurrent CeAD remained unstudied. The recruitment of the patients in stroke centers may have biased the sample toward overrepresentation of patients with ischemic first events [18]. This bias may have 
affected the frequency of ischemic recurrent events also, due to the observation of this study that ischemia upon the first CeAD increased the risk for ischemia upon a later event. This secondary effect of the biased recruitment of patients with first CeAD events implies that the observed frequency of stroke or TIA due to recurrent CeAD events might also be inflated. In the study, seven of 76 recurrent events caused stroke and six caused TIA. These figures are probably overestimations, due to biased sampling. Vascular imaging of patients with an acute CeAD sometimes suggests that an earlier CeAD event had occurred that apparently remained undiagnosed. In the current study, we did not systematically search for such patients with a history of asymptomatic previous CeAD events. Notwithstanding, the finding of asymptomatic previous events is interesting: it suggests that second dissection must not be milder than first dissections. It is well conceivable that the detection of CeAD events by vascular imaging allowed the diagnosis of many events with very mild symptoms, whereas most patients with such mild symptoms don't seek medical advice, resulting to underrepresentation of these patients in hospital-based registries. In a recent study of serial ultrasound examinations of CeAD patients, five recurrent events were detected. In all patients with recurrent CeAD, new symptoms were reported [19]. These included newly occurring or new characteristics of headache $(n=5)$ with concomitant neck pain $(n=3)$ not reported before, or with pulsatile tinnitus $(n=2)$. Although the number of recurrent CeAD events in this study was small, the absence of clinically silent events may be related to the high frequency of ultrasound examinations performed. We speculate that some minor symptoms, like changes in the characteristics of headache may be not reported by some of the patients of the current study, due to a possibly longer delay between the minor symptoms and the vascular imaging, which was performed at the end of the 3-6 months follow-up period.

In conclusion, recurrent CeAD typically affects the same site of artery, usually on the contralateral side. Recurrent CeADs seem to cause ischemic events less often than initial CeADs. The risk that patients who presented with solely non-ischemic symptoms for an initial CeAD, will have ischemic symptoms in case of a recurrent CeAD seems very small.

\section{Disclosures}

None.

\section{Funding:}

None. 


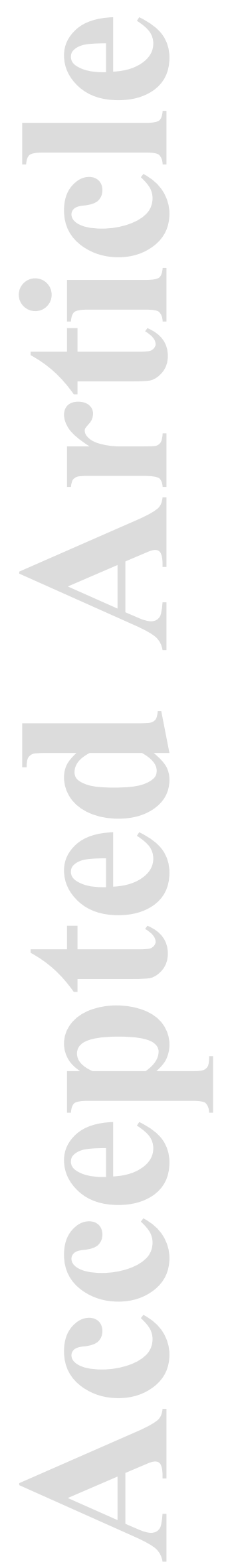

This article is protected by copyright. All rights reserved 


\section{References}

1. Debette S, Leys D. Cervical-artery dissections: predisposing factors, diagnosis, and outcome. Lancet Neurol 2009; 8: 668-678.

2. Brott TG, Halperin JL, Abbara S, et al.

\section{ASA/ACCF/AHA/AANN/AANS/ACR/ASNR/CNS/SAIP/SCAI/SIR/SNIS/SVM/SVS}

guideline on the management of patients with extracranial carotid and vertebral artery disease: executive summary. A report of the American College of Cardiology Foundation/American Heart Association Task Force on Practice Guidelines, and the American Stroke Association, American Association of Neuroscience Nurses, American Association of Neurological Surgeons, American College of Radiology, American Society of Neuroradiology, Congress of Neurological Surgeons, Society of Atherosclerosis Imaging and Prevention, Society for Cardiovascular Angiography and Interventions, Society of Interventional Radiology, Society of Neuro-interventional Surgery, Society for Vascular Medicine, and Society for Vascular Surgery. Circulation 2011; 124: 489-532.

3. Morris NA, Merkler AE, Gialdini G, Kamel H. Timing of Incident Stroke Risk After Cervical Artery Dissection Presenting Without Ischemia. Stroke 2017; 48: 551-555.

4. Engelter ST, Traenka C, Lyrer P. Dissection of Cervical and Cerebral Arteries. Curr Neurol Neurosci Rep 2017; 17: 59.

5. Dittrich R, Ritter MA, Ringelstein EB. Ultrasound in spontaneous cervical artery dissection. Perspectives in Medicine 2012; 1: 250-254.

6. Debette S. Pathophysiology and risk factors of cervical artery dissection: what have we lea rnt from large hospital-based cohorts? Curr Opin Neurol 2014; 27: 20-28.

7. Compter A, Schilling S, Vaineau CJ, et al. Determinants and outcome of multiple and early recurrent cervical artery dissection. Neurology 2018; 91: e769-e780.

8. Dittrich R, Nassenstein I, Bachmann R, et al. Polyarterial clustered recurrence of cervical artery dissection seems to be the rule. Neurology 2007; 69: 180-186.

9. Kloss M, Grond-Ginsbach C, Ringleb P, Hausser I, Hacke W, Brandt T. Recurrence of cervical artery dissection: an underestimated risk. Neurology 2018; 90: e1372-e1378.

10. Guglielmi V, Visser J, Arnold M, et al. Triple and quadruple cervical artery dissections: a systematic review of individual patient data. J Neurol 2019; 266: 1383-1388.

11. Traenka C, Grond-Ginsbach C, Goeggel Simonetti B, et al. Artery occlusion independently predicts unfavorable outcome in cervical artery dissection. Neurology 2020; 94: e170-e180. 
12. Morris DR, Ayabe K, Inoue T, et al. Evidence-based carotid interventions for stroke prevention: state-of-the-art review. J Atheroscler Thromb 2017; 24: 373-387.

13. Huibers A, Calvet D, Kennedy F, et al. Mechanism of procedural stroke following carotid endarterectomy or carotid artery stenting within the International Carotid Stenting Study (ICSS) randomised trial. Eur J Vasc Endovasc Surg 2015; 50: 281-288.

14. Wieker CM, Harcos K, Ronellenfitsch U, Demirel S, Bruijnen H, Böckler D. Impact of routine completion angiography on outcome after carotid endarterectomy. J Vasc Surg 2019: 69: 824831.

15. Rothwell PM. Endarterectomy for symptomatic and asymptomatic carotid stenosis. Neurol Clin 2008; 26: 1079-1097.

16 Martin JJ, Hausser I, Lyrer P, et al. Familial cervical artery dissections: clinical, morphologic, and genetic studies. Stroke 2006 ;37: 2924-2929.

17 Chen CJ, Green IE, Worrall BB, Southerland AM. Cerebral collaterals and stroke in patients with isolated carotid artery dissections. J Clin Neurosci 2020; 72: 158-162.

18. Grond-Ginsbach C, Metso TM, Metso AJ, et al. Cervical artery dissection goes frequently undiagnosed. Med Hypotheses 2013; 80: 787-790.

19. Traenka C, Streifler J, Lyrer P, Engelter ST. Clinical Usefulness of Serial Duplex Ultrasound in Cervical Artery Dissection Patients. Cerebrovasc Dis. 2020; 49: 206-215 


\section{Legends to the tables:}

Table 1. Differences and correspondences between first and subsequent CeAD events. Table $1 \mathrm{~A}, 1 \mathrm{C}$ and $1 \mathrm{E}$ compares the sites of $\mathrm{CeAD}$, the vascular imaging findings and the symptoms between the 76 first and the 76 second CeAD events of the study sample. The $3 \times 3$ contingency tables $(1 \mathrm{~B}, 1 \mathrm{D}, 1 \mathrm{~F})$ show the number of patients with combined characteristics of the first and the second event. ICA: internal carotid artery; VA: vertebral artery. P-values of Fisher exact tests, multiplied by 6 to correct for multiple testing.

\section{Table 2. Predictors of ischemia due to cervical artery dissection.}

CeAD, cervical artery dissection; SD, standard deviation; ICAD, internal carotid artery dissection; VAD, vertebral artery dissection; CI, confidence interval. Multivariate p-values and odd ratios were calculated in a logistic regression model with ischemia as dependent variable. All other variables were entered simultaneously as predictors. The model identified independent associations with ischemia among the $152 \mathrm{CeAD}$ events of the study sample. 


$\begin{array}{lllll}\text { first } & \text { second } & & \text { first } x \text { second } & \\ \text { event } & \text { event } & \text { p-value } & \text { event } & \text { p-value }\end{array}$

Location of CeAD

ICA

$V A$

Both

\section{Vascular imaging}

Normal lumen

Stenosis

Occlusion
A

0.469

\begin{tabular}{|l|l|l|l|}
\hline 32 & 5 & 0 & 37 \\
\cline { 1 - 2 } 15 & 20 & 1 & 36 \\
\cline { 1 - 2 } 3 & 0 & 0 & 3 \\
\cline { 1 - 2 } 50 & 25 & 1
\end{tabular}

0.0008

\begin{tabular}{|c|c|c|c|c|}
\hline 5 & 10 & 4 & 19 & \\
\hline 2 & 30 & 24 & 56 & 0.080 \\
\hline 0 & 0 & 1 & 1 & \\
\hline
\end{tabular}




\section{Symptoms}

No symptom

Local symptoms

$0 \quad 39$

Ischemia

$34 \quad 24$

$<0.0001$

\begin{tabular}{|l|l|l|l|}
\hline 0 & 17 & 22 & 39 \\
\cline { 1 - 3 } 0 & 16 & 8 & 24 \\
\cline { 1 - 3 } 0 & 1 & 12 & 13 \\
\cline { 1 - 3 } 0 & \multicolumn{2}{|c|}{34} & \multicolumn{2}{|c}{42} &
\end{tabular}

0.012

$42 \quad 13$

Table 1. Differences and correspondence s between first and subsequent CeAD events.

\begin{tabular}{|c|c|c|c|c|c|c|}
\hline & no & ischemic & univariate & multivariate & & Odd ratio \\
\hline & ischemia & $\mathrm{CeAD}$ & $\mathrm{p}$ & $\mathrm{p}$ & & {$[95 \% \mathrm{CI}]$} \\
\hline & $(n=97)$ & $(\mathrm{n}=55)$ & & & & \\
\hline Female sex & $49(50.5)$ & $31(56.4)$ & 0.504 & 0.993 & 1.004 & {$[0.379-2.541]$} \\
\hline Age & $40.7 \pm 9.3$ & $40.3 \pm 8.3$ & 0.777 & 0.321 & 1.004 & {$[0.379-2.541]$} \\
\hline
\end{tabular}

This article is protected by copyright. All rights reserved 


\section{ICAD}

multi-vessel CeAD

open/stenotic/occluded vessel

second event
$56(57.7)$

$5(5.2)$

$25 / 68 / 4$

$63(64.9)$
$35(63.6)$

$7(12.7)$

$1 / 28 / 26$

$13(23.6)$
0.496

0.121

$<0.001$

$<0.001$
0.788

0.120

$<0.001$

0.028
$0.877 \quad[0.369-2.131]$

$3.298 \quad[0.734-14.812]$

10.693 [3.754-30.458]

$0.373 \quad[0.155-0.898]$

Table 2. Predictors of ischemia due to cervical artery dissection. 